\title{
Electrically driven ferroelastic domain walls, domain wall interactions, and moving needle domains
}

\author{
Guangming Lu, ${ }^{1,2}$ Suzhi Li, ${ }^{1, *}$ Xiangdong Ding, ${ }^{1, \dagger}$ Jun Sun, ${ }^{1}$ and Ekhard K. H. Salje $\oplus^{1,2, \sharp}$ \\ ${ }^{1}$ State Key Laboratory for Mechanical Behavior of Materials, Xi'an Jiaotong University, Xi'an 710049, China \\ ${ }^{2}$ Department of Earth Sciences, University of Cambridge, Cambridge CB2 3EQ, United Kingdom
}

(Received 7 June 2019; revised manuscript received 26 August 2019; published 8 November 2019)

\begin{abstract}
Ferroelastic domains generate polarity near domain walls via the flexoelectric effect. Applied electric fields change the wall dipoles and generate additional dipoles in the bulk. Molecular dynamics simulations show that the thickness of domain walls changes when an electric field is applied to the sample. Fields parallel to the walls lead to expansion of the wall thickness while fields perpendicular to the wall lead to shrinking of the wall thickness. The interactions between polar domain walls expand over more than 45 unit cells, the resulting forces change the wall-wall distances if pinning effects are small. The interaction increases nonlinearly with decreasing wall-wall distances favoring equal wall distances as the consequence of energy minimization under the constraints of a constant number of domain walls. Even for small groups of three walls the sequence of walls is locally periodic: assemblies of three parallel domain walls arrange themselves so that the intermediate domain wall is located exactly in the middle between the two outer walls. The driving force is appreciable if the distance between the outer domain walls is below approximately 30 lattice units. Pairs of domain walls often form needle domains where the shaft of the needle is ca. 3 lattice units wide. The movement of needle domains under applied electric field was simulated. The advancement and retraction of needles is larger in finite samples with charge-free surfaces than under periodic boundary conditions in the bulk. The needle tip moves even more freely when the sample surface is charged.
\end{abstract}

DOI: 10.1103/PhysRevMaterials.3.114405

\section{INTRODUCTION}

Ferroelasticity is defined by the ferroelastic hysteresis [1] where the dominant hysteretic process is the progression and retraction of needle domains [2-12]. Needle domains do indeed dominate most ferroelastics, although there are a few cases where this is not the case. The dynamics of the switching process leads to avalanche mechanisms where each switch has a high probability to induce secondary switches $[6,13-$ 18]. These switches are largely athermal because the energy barriers between different domain states are usually much greater than the thermal energy. It was shown that ferroelastic domain boundaries are generally polar [19-25]. The first example was $\mathrm{CaTiO}_{3}$ [19] where polarity was observed in transmission electron microscopy. This observation was further confirmed by other techniques, such as second harmonic generation (SHG) $[20,23,26,27]$. Polarity was also predicted on theoretical grounds [24]. Experimentally, it was found that virtually all ferroelastic domain walls contain some polarity [25] and hence a new question arose: Can a ferroelastic needle domain be switched by an electric field, rather than by strain? Gao et al. [28] argued that in thin film ferroelectric devices, switching of ferroelastic domains significantly enhances the electromechanical response. Switching dynamics of individual ferroelastic domains in thin $\mathrm{Pb}\left(\mathrm{Zr}_{0.2} \mathrm{Ti}_{0.8}\right) \mathrm{O}_{3}$ films under electrical and mechanical excitations was stabilized by

\footnotetext{
*lisuzhi@xjtu.edu.cn

†dingxd@mail.xjtu.edu.cn

‡ekhard@esc.cam.ac.uk
}

dislocations at the substrate interface [28]. For both electrical and mechanical switching, ferroelastic switching seems to occur most readily at the highly active tips of ferroelastic needle domains. Such switching of ferroelastic domains in ferroelectric $\mathrm{BaTiO}_{3}$ was observed in low energy electron microscope (LEEM) experiments [29]. Electric switching of 90 degree domains in $\mathrm{BaTiO}_{3}$ follows the same patterns as purely ferroelastic switching [30]. The predominance of strain induced (ferroelastic) switching over electric field induced (ferroelectric) switching is expected because the elastic degrees of freedom carry substantially more energy than the electric component [7].

In a different scenario, the observed electric movement of needle domains in $\mathrm{SrTiO}_{3}$ was shown to stem mainly from the dielectric anisotropy which expands or shrinks a domain [31] so that one might conclude that electric switching requires either dielectric anisotropy or the rapid formation of dislocations. To test this hypothesis we used a dielectrically isotopic, ferroelastic toy model to show that a needle domain can also be moved by an electric field even when the dielectric response is isotropic. Furthermore, needle domains may change their internal structure under an electric field because the coupling (biquadratic or gradient coupling) between strain and polarization will change when one component is constrained by an external field [32-36]. We use molecular dynamics simulations to confirm this effect in a simple toy model. An applied electric field in "purely" ferroelastic materials with no polarity in the bulk also changes the internal properties of domain walls and their interactions. A change of the wall thickness changes the wall energy and we show in this paper that such changes are significant. Interactions between twin 
TABLE I. The interaction potential in binary system. For different ranges of nearest neighbor $(\mathrm{NN})$ interactions, we set different forms of potential function to describe them. Besides the short-range interactions, the long-range Coulomb forces were considered.

\begin{tabular}{lccc}
\hline \hline Interactions & Range & & Potential form \\
\hline & & First NN & $20(r-1)^{2}$ \\
A-A & Short-range & Second NN & $-10(r-\sqrt{2})^{2}+8000(r-\sqrt{2})^{4}$ \\
& & Third NN & $8(r-3)^{4}$ \\
& Long-range & Fourth NN & $-10(r-\sqrt{5})^{2}+5100(r-\sqrt{5})^{4}$ \\
B-B & Short-range & - & Coulomb interaction, dielectric constant $=1000$ \\
& Long-range & First NN & $20(r-1)^{2}$ \\
A-B & Short-range & - & $1.5(r-\sqrt{2})^{2}$ \\
& Long-range & First NN & Coulomb interaction, dielectric constant $=1000$ \\
\hline \hline
\end{tabular}

walls are equally impacted by changes of the internal wall structure and the orientation of induced dipoles in the walls and in the bulk. These changes relate to the compactification of multiple well potentials [37] to minimizers that generally depend on the internal structure of the wall when several order parameters are activated to generate a domain wall [32,33,38,39]. An early approach [40] to introduce explicit wall-wall interactions was to consider overlapping wall profiles of parallel domain walls. This interaction was formulated to be exponential and repulsive. It was assumed to extend only over very few lattice units corresponding to direct strainstrain interactions. Unexpectedly, Bratkovsky and Levanyuk [41] found no such contributions to the dielectric response by interacting domain walls and no ordering within their analytical theory. Here we show that both the short range elastic interaction exist as also much more long-ranging interactions between domain walls when the dipolar nature of the domain wall is taken into account. We report interaction distances well beyond 25 unit cells, which amounts to typical wall distances of some $125 \mathrm{~nm}$. This result agrees perfectly with recent observations by Everhardt et al. [42] who reports domain wall interactions for distances of $>100 \mathrm{~nm}$.

\section{ATOMIC MODEL}

Molecular dynamic simulations are based on a twodimensional toy model with two base atoms (A and B) carrying opposite charges [25]. This model is based on simple nonlinear elastic interactions (Landau springs) and harmonically coupled displacements of charged particles [40,43,44]. The potential of the cation $\mathrm{A}$ and anion $\mathrm{B}$ sublattices are kept the same as in Ref. [27]. The interaction between the A-B sublattices was strengthened to produce a stronger coupling effect between strain and charge, mimicking the electric field driven effects (piezoelectricity or electrostriction) in real materials $[21,45,46]$. The model parameters are inspired by $\mathrm{SrTiO}_{3}$ with the energy scale determined by $T_{c}=105 \mathrm{~K}$, a typical ferroelastic shear angle of $2^{\circ}$, and wall dipoles related to displacements of $6 \mathrm{pm}$ as observed in $\mathrm{CaTiO}_{3}$ [19]. All modified model parameters are summarized in Table I.

The polar displacement in each unit lattice is defined as the displacement of anion with respect to the center position of its four cation neighbors. The direction points from anion to the averaged position of cations. We then determine the polarization $P=q s / V$, where $q$ is the electron charge, $s=$ $\sqrt{s_{x}^{2}+s_{y}^{2}}$ is the polar displacement for each unit cell, and $V$ is the volume of the unit cell.

We compute the ferroelastic nanostructures using the large-scale atomic/molecular massively parallel simulator (LAMMPS). First, the internal structure of domain walls and their distances for pairs of wall, such as in the shafts of needle domains, were simulated under periodic boundary conditions. The relevant simulations were carried out under a fixed number of particles, temperature, and pressure, known as the isothermal-isobaric ensemble [47,48]. The sample lengths in the $x$ and $y$ directions depend on the applied field due to electrostrictive and piezoelectric effects. The simulation box contains four domain walls at different interwall distance. One pair of domain walls is separated by three layers of the $\mathrm{A}$ lattice and four layers of the B lattice. A second pair contains a spacer of five layers of the A lattice and six layers of the B lattice. One solitary domain wall is located at the top of the simulation box. This configuration was constructed to investigate a single domain wall and multi-wall interactions (where the interwall distances were adjusted). The direct interactions between two domain walls with varies interwall distances were investigated by using sandwich configurations under periodic boundary conditions [6].

In a separate simulation, we investigated the movement of needles driven by the direct coupling between external electric field and wall dipoles under periodic boundary conditions. The relevant simulations were based on the same isothermalisobaric ensemble $[47,48]$. Finally, open boundary conditions were used to simulate the movement of needles including surface effects. We use one charge-free configuration and one charged surface configuration. The charge-free configuration possesses surface layers with an equal number of $\mathrm{A}$ and $\mathrm{B}$ atoms [49]. The surface layer is the crystallographic plane (11) and the needle domain is declined by ca. 45 degrees from the surface, i.e., the needle direction is [01]. We apply an electric field along, perpendicular and under 45 degree inclined to the needle direction, namely, along [01], [10], and [11]. A charged surface configuration was constructed to mimic a two-dimensional thin film assembly in which the 

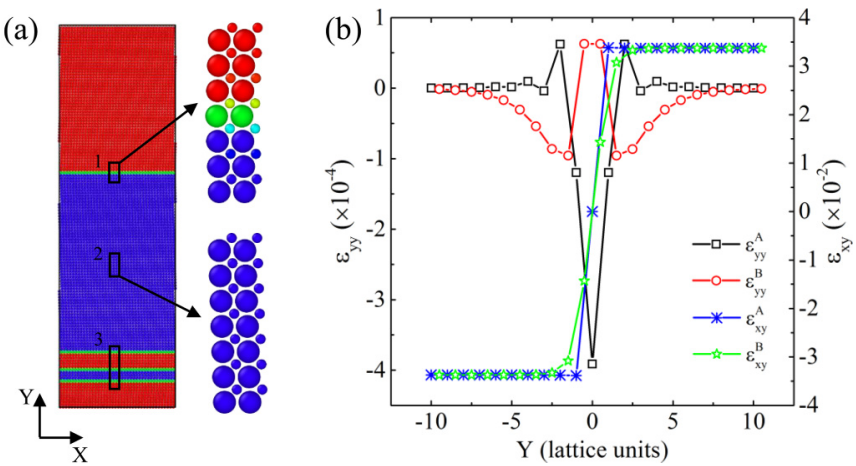

FIG. 1. (a) The model domain structure consists of one solitary wall (indicated by "1") and three close walls (indicated by "3"). The atoms are colored according to the local shear strain $\varepsilon_{x y}$. (b) The strain profiles of the lone wall for A and B sublattices. The shear strain $\varepsilon_{x y}$ follows the usual tanh-profile while the strains in the $y$ direction $\varepsilon_{y y}$ show a complex profile like a breather [1].

charged surface can survive due to the electrostatic effect. The left and bottom surfaces have A atom terminations, while the upper and right surfaces have B atom terminations. Such configurations may also be observed in bulk materials on a fairly local scale [50].

\section{RESULTS AND DISCUSSION}

\section{A. Single domain walls}

We created a simulation box with 40 lattice units in the $x$ direction and 132 lattice units in the $y$ direction, as shown in Fig. 1(a). It contains a single domain wall (indicated by "1") and three twin walls with smaller distances (indicated by "3"). Electric dipoles appear inside the domain wall and are aligned parallel to the wall. Additional small canted dipoles occur in adjacent layers. A similar configuration was found experimentally in $\mathrm{CaTiO}_{3}[19,51]$. The combination of displacement vectors defines the polarization pattern in and near the wall. The A and B sublattices are heavily strained at the domain wall. The strains are defined by the ferroelastic order parameter $\varepsilon_{x y}$ and a secondary order parameter $\varepsilon_{y y}$. The profiles are shown in Fig. 1(b), where $\varepsilon_{x y}$ follows the classic tanh-profile while $\varepsilon_{y y}$ displays a breather with a much broader profile than $\varepsilon_{x y}$ [1].

Electrical fields were then applied with $E_{x}$ along [10], $E_{x y}$ along [11], and $E_{y}$ along [01]. The applied electric field was held constant for $70 \mathrm{ps}$ at each step while the structure was allowed to relax. This time is much longer than the characteristic phonon time of the bulk material at $T=0.001 \mathrm{~K}$, namely, $\tau_{\text {phonon }}=3.85$ ps. A bulk area [indicated by "2" in Fig. 1(a)] contains the same number of atoms and was recorded for comparison with the wall region. The electric field shifts the A and B sublattices against each other. These shifts lead to small structural changes in the wall when an electric field $E_{x}$ is applied. The field-induced strain $\left(\varepsilon_{y y}\right)$ is in the order of magnitude $10^{-4}$. Figure 2(a) shows the averaged lattice parameters $\langle\mathrm{b}\rangle$ along the $y$ direction (expressed as lattice repetition unit, which is defined as the repetition unit in the undisturbed bulk).

The wall thickness was measured by the lattice repetition length between adjacent four layers across the wall and (a)

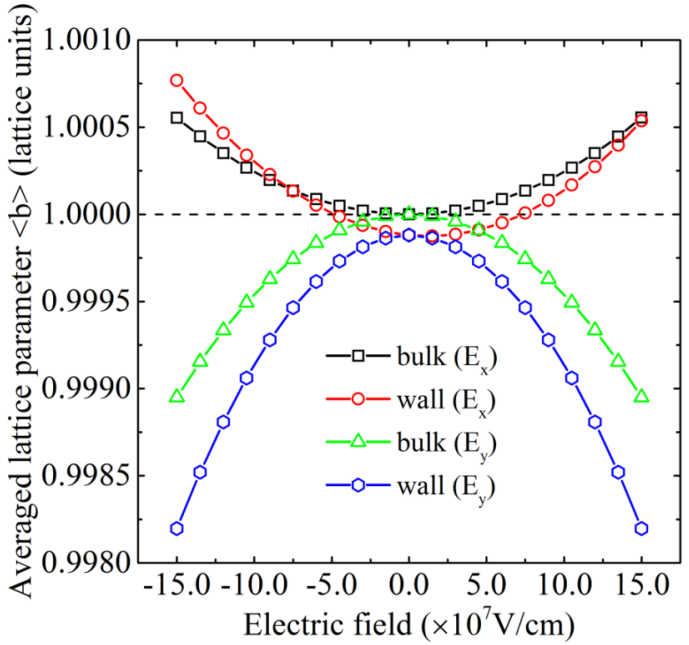

(b)

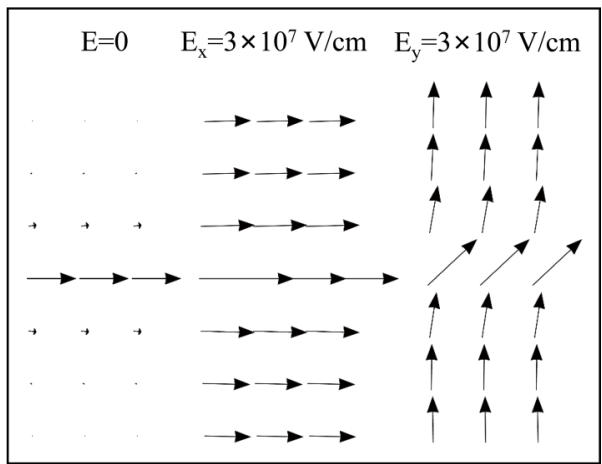

FIG. 2. (a) Averaged lattice parameter $\langle b\rangle$ as a function of the external electric field. (b) The dipole configurations of the lone wall under fields along [10] and [01] orientations. The applied field $E_{x}$ leads to an expansion of the domain wall, while the field $E_{y}$ leads to the contraction of the domain wall.

was found to be smaller than in the bulk for $E_{y}$ and $E_{x y}$ but not for $E_{x}$. The change of the repetition units can be rationalized in the bulk by parallel, induced dipoles generating repulsive forces [Fig. 2(b)] and a positive (expanding) electrostrictive effect. Near domain walls, the interactions are more complex. An electrostrictive effect is superposed by a weak piezoelectric effect [Fig. 2(a)]. The overall trend is the expansion of the domain wall width by an applied field $E_{x}$. In contrast, a field $E_{y}$ contracts the domain wall width following a head-to-tail configuration of the induced dipoles [Fig. 2(b)]. The field $E_{x y}$ also contracts the domain wall width and shows negative electrostriction in the wall and bulk, as shown in Fig. S1 [52]. This electric field also changes the wall structure where the tanh-profile of the primary strain order parameter is initially accompanied by a breather profile of wall lattice parameters under an applied field $E_{x}$ [1]. Strong breather-type singularities are found for $\varepsilon_{y y}$. This profile is similar to those found in surface strain simulations $[49,53]$. The breather flattens with increasing field $E_{y}$. The thickness of the domain wall decreases accordingly. Note that the thickness of the domain walls ( $\sim 4$ lattice units) depends on the model parameters. Nevertheless, the fact that the domain wall thickness is field dependent was found in all simulations. 

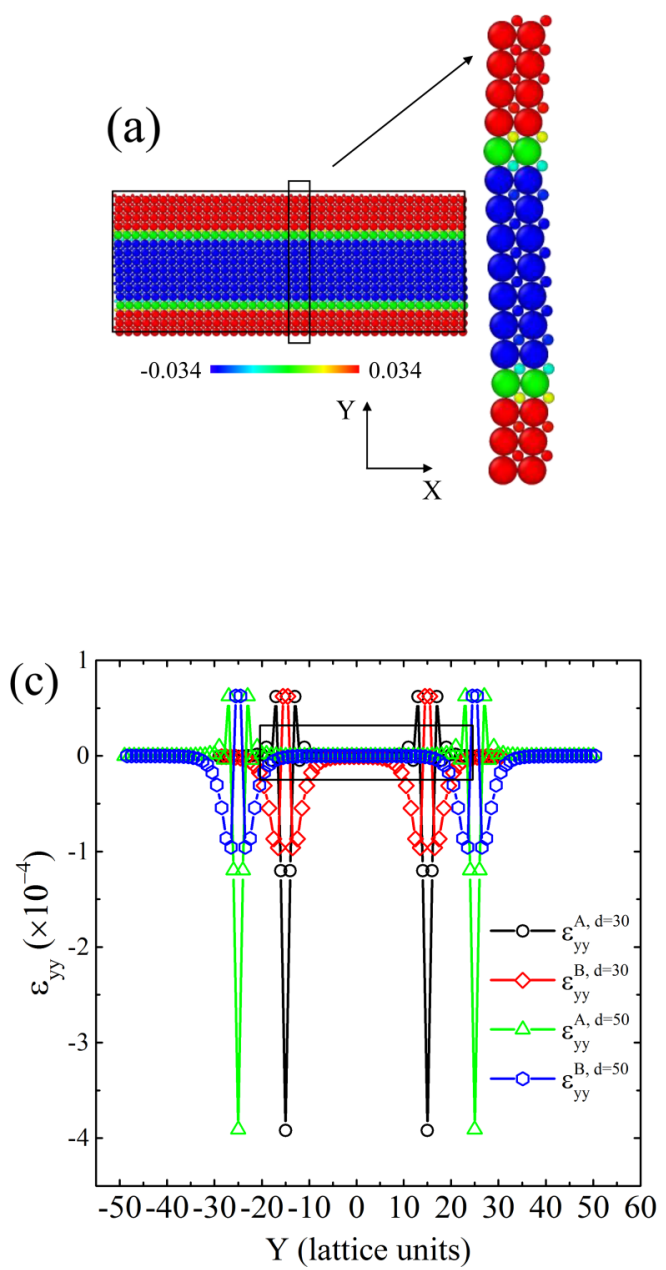

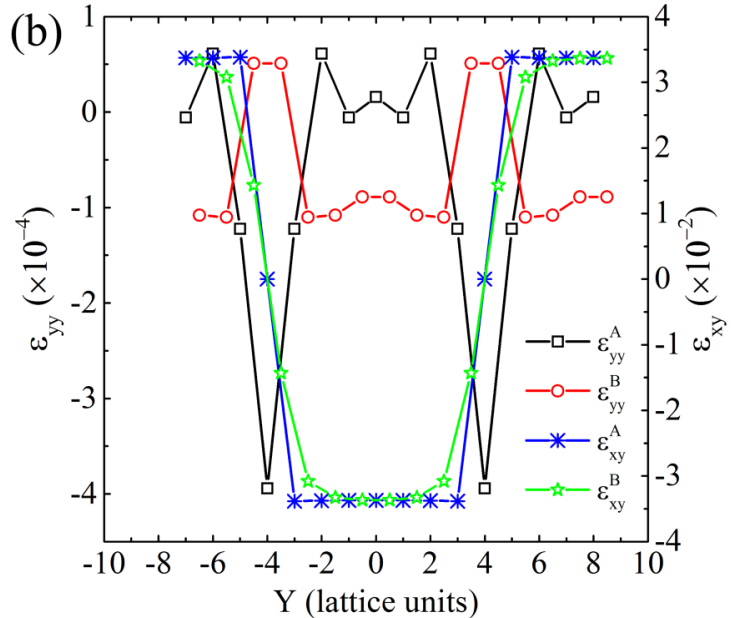

(d)

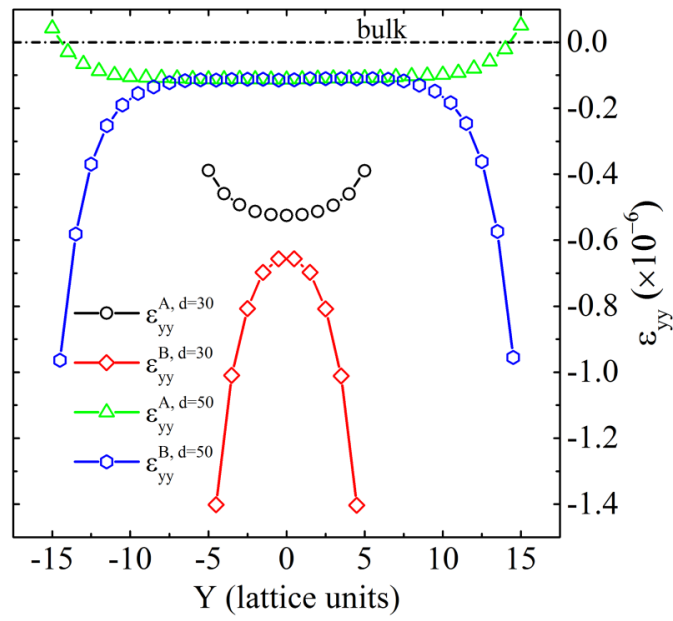

FIG. 3. (a) Domain structure consists of two interactive twin walls. The atomic asymmetric structure is colored according to the atomiclevel shear strain $\varepsilon_{x y}$. (b) The overlapping strain profiles of a wall pairs with $d=8$ lattice units. (c) A lattice contraction is caused by the attractive forces of the antiparallel wall dipole arrays ( $d=30$ lattice units and 50 lattice units). (d) The local area indicated by the black rectangular in (c). The magnitude of local strain is on order of $\sim 10^{-7}$.

\section{B. Wall-wall interactions}

Wall-wall interactions were simulated under periodic boundary conditions. The single twin structure in Fig. 1(a) is now replaced by pairs of twin planes with constant length (40 lattice units) in the $x$-axis direction and variable inter wall distances $d$ (3 lattice units $<d<50$ lattice units) in the $y$-axis direction. Figure 3(a) shows an example with $d=8$ lattice units. Direct wall interactions due to the overlapping strain of the B sublattice are observed [Fig. 3(b)]. This interaction reduces the interwall distances including the change of the wall thickness. Figs. 3(c) and 3(d) shows the contraction of the wall pairs caused by the interaction of two antiparallel dipole arrays residing inside the twin walls. The interwall distances $(d=30,50$ lattice units) are already quite large and beyond the expected overlap length of two isolated walls (see Fig. 4). The lattice contraction beyond the overlapping regions of the two solitary walls stems from the attractive forces of the two antiparallel dipole arrays of the two domain walls. This local contraction is small $\left(\varepsilon_{y y} \sim 10^{-7}\right)$ [Fig. 3(d)] compared with the contraction induced by the overlapping strains $\left(\varepsilon_{y y} \sim 10^{-4}\right)$.
We now describe the interaction energy of the two parallel domain walls (Fig. 4). The total energy depends on the system size. The systems have the same dimension in the $x$ direction and vary in the $y$ direction. In the case of noninteracting walls the energy is a simple superposition of the wall and the bulk energy. The averaged bulk potential energy is $-27.41 \mathrm{meV}$ per atom. The averaged excess energy of two polar walls with respect to the bulk is $1.86 \mathrm{meV}$ per atom, which corresponds to some $1.19 \times 10^{-9} \mathrm{~mJ} / \mathrm{m}$ in perovskite. This value is close to wall energies estimated in three-dimensional structures, which is $20 \mathrm{~mJ} / \mathrm{m}^{2}$ by taking into account the thickness of the sample of ca. $10^{-10} \mathrm{~m}$ [54-56]. The equivalent potential energy for the bulk and nonpolar walls (when the charges in our model are set to zero) is $10.95 \mathrm{meV}$. The excess energy of two nonpolar walls is $1.87 \mathrm{meV}$ per atom, which corresponds to some $1.19 \times 10^{-9} \mathrm{~mJ} / \mathrm{m}$ in perovskites. The dependence of the potential energy is shown for both scenarios in Figs. 4(a) and 4(b). We now consider a fixed system size comprising rows of $121 \mathrm{~A}$ atoms and $121 \mathrm{~B}$ atoms in the $y$ direction. Within this system we separate the twin walls between 8 and 45 lattice units. For each configuration we calculate 
(a)
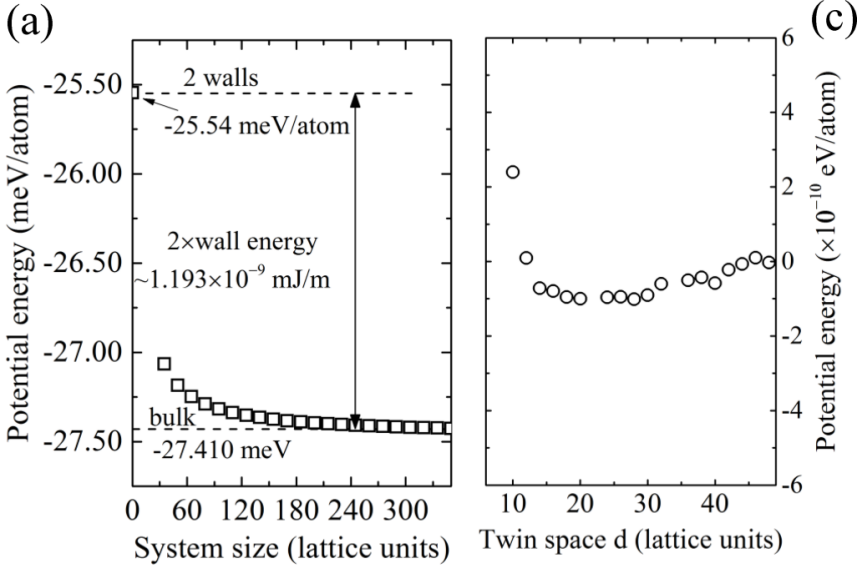

(b)

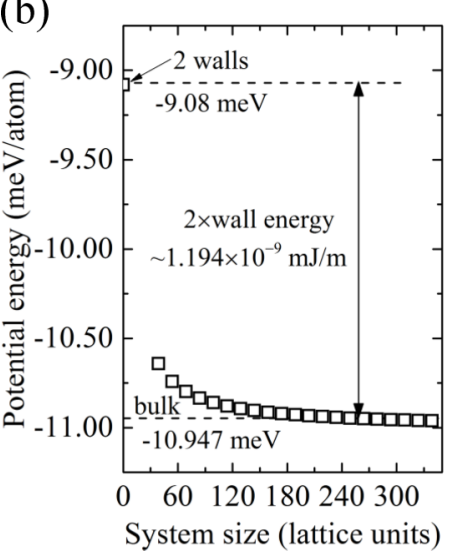

(c)

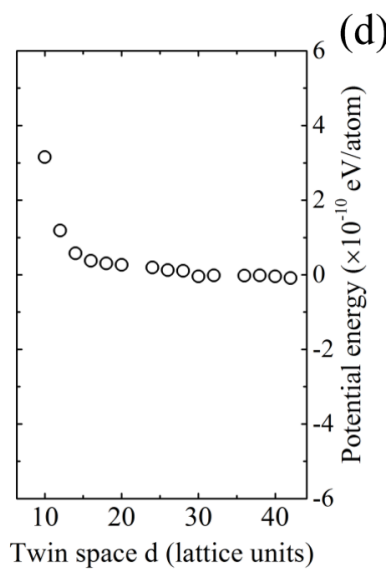

FIG. 4. The potential energy per atom of the twin pair in a matrix as a function of the system size perpendicular to the walls for (a) polar walls and (b) nonpolar walls. For a fixed system size (around 120 atoms in the $y$ direction), the potential energy changes as a function of the twin distance for (c) polar walls and (d) nonpolar walls. The polar twin walls have repulsive force for distances $d<25$ lattice units and attractive force for $d>25$ lattice units. There is no such attractive interaction for nonpolar walls.

the potential energy. In noninteracting systems, the resulting potential energy is independent of the interwall distance. Figures 4(c) and 4(d) show that this is only the case for the largest interwall distances. For small distances we find a steep exponential decay followed by a weak attractive potential in case of polar walls. In the case of nonpolar walls, we find only the exponential decay. The interesting feature is that the repulsive short-range interaction extends to approximately 20-30 lattice units in the nonpolar system. In the polar system we find an energy minimum near 25 lattice units. For larger distances we find a weak attractive dipole-dipole interaction. The corresponding equilibrium distance is hence near 25 lattice units, which corresponds to approximately $125 \mathrm{~nm}$ in a typical perovskite structure. The field dependence of the pair interaction is shown in Fig. 5. This result is crucial for the understanding of wall-wall interactions. Elastic interactions lead to repulsion over very small distances while the additional dipole moments in domain walls lead to much longer interactions and a shallow energy minimum which is the origin of an "intrinsically" stable interwall distance.

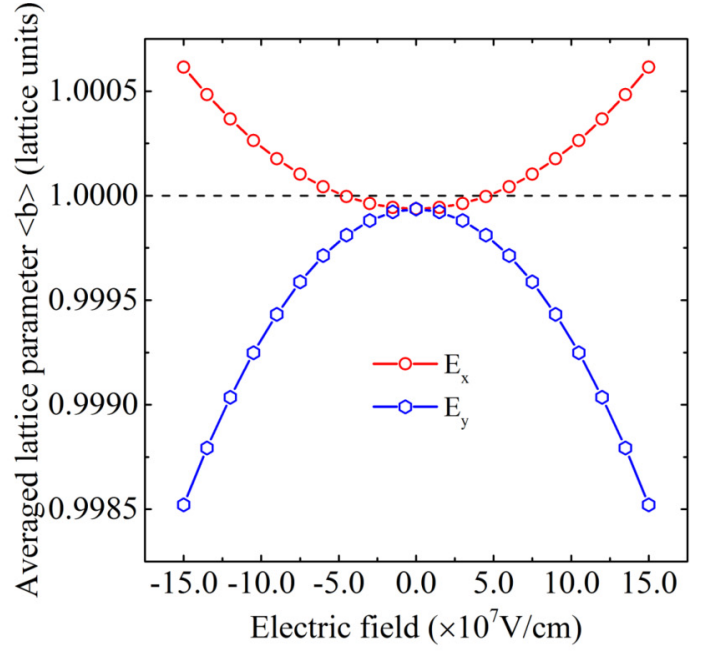

(b)

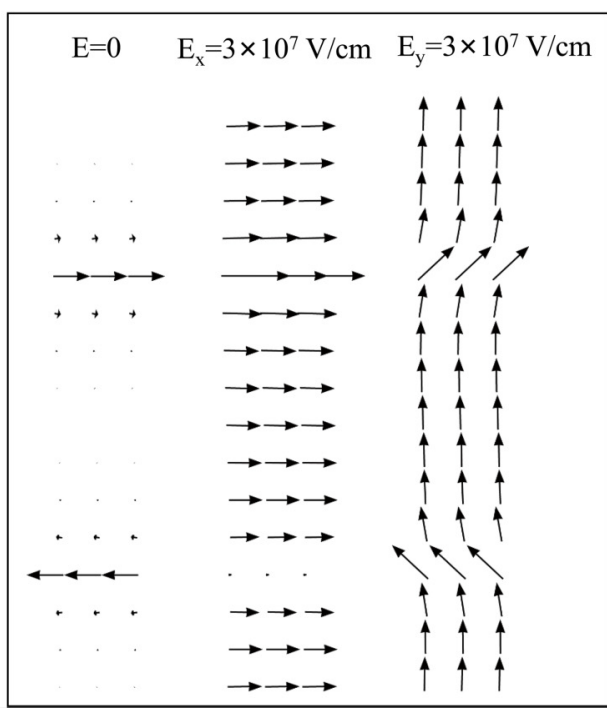

FIG. 5. (a) Averaged lattice parameter $\langle b\rangle$ as a function of the external electric field for a pair of domain walls with the wall-wall distance of 8 lattice units. (b) The dipole configurations of wall under fields in the [10] and [01] directions. The unit length is defined as the repetition length in the bulk with no twin boundaries. The fieldfree state shows a slightly reduced lattice unit because of the wallwall interaction. Fields lead to electrostrictive expansion $\left(E_{x}\right)$ and contraction $\left(E_{y}\right)$.

The interaction of pairs of domain walls demonstrates that the "primary" order parameter, namely the shear strain follows simple tanh-profiles and is hardly influenced by the wall-wall interaction while the "secondary" order parameter of the compressive strain perpendicular to the twin wall shows a complex behavior. The profile of the domain wall is greatly modified, first with respect to the bulk structure and, second, with respect to the wall-wall interaction. This leads to a contraction of the wall thickness and of the bulk that is sandwiched between the walls. The energy profile in Fig. 4(c) shows that the wall-wall interaction is repulsive for short distances and attractive for larger distances. For short distances this shows the predicted exponential repulsion, which stems from the overlap between the walls and the resulting higher density 
(a)

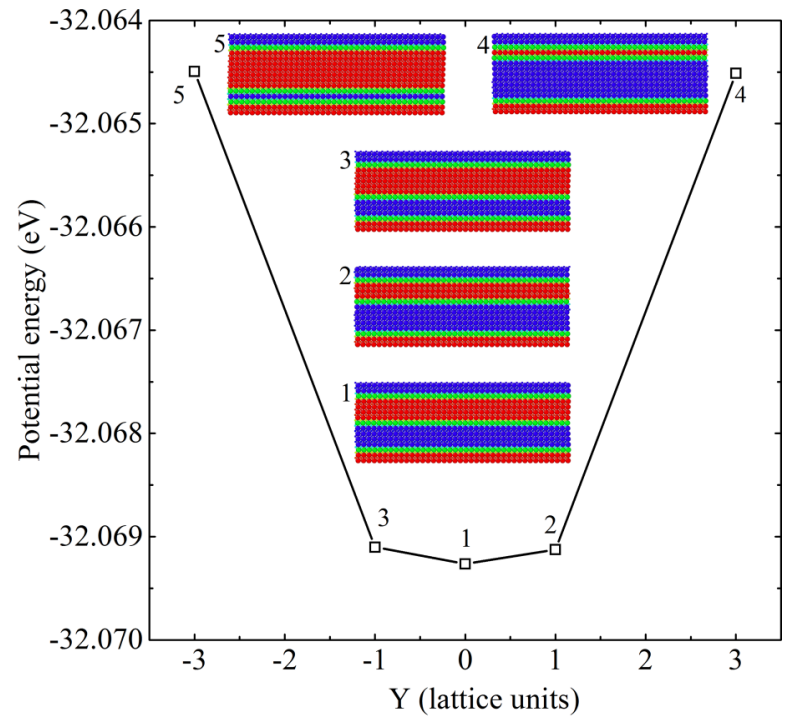

(b)

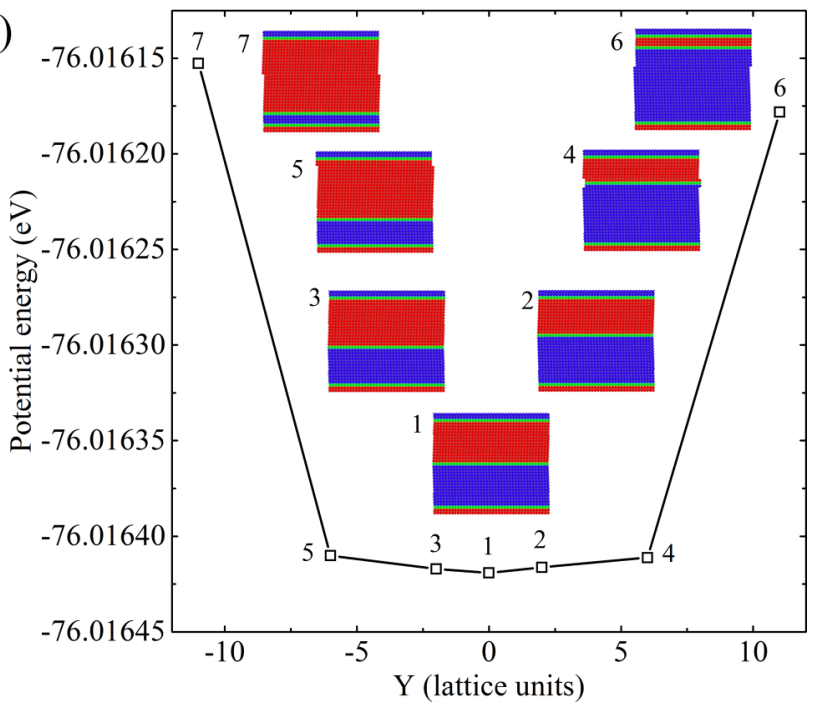

FIG. 6. The variation of potential energy as the function of the position of inner domain wall, while the positions of two outer domain walls are kept the same. The distance between two outer walls are (a) 10 lattice units and (b) 30 lattice units. The system has the lowest energy when the inner domain wall is right in the middle. The colors of atomic images are coded according to the atomic-level shear strain $\varepsilon_{x y}$. The red and blue regions represent two oriented domains and the green layer represents the domain wall.

of the local structure. This higher density leads to excess energies, which repel the two walls.

\section{Multi-wall interactions and the sideways movement of domain walls}

The interwall interaction leads to specific array patterns, most commonly needle domains with equal distance when the interwall distances are sufficiently small. We now explore the origin of the equidistant configuration by keeping two outer domain walls at a constant distance while a third inner domain wall is allowed to move sideways. This is the configuration investigated by Everhardt et al. [43]. We choose $d=10$ lattice units [Fig. 6(a)] and 30 lattice units [Fig. 6(b)] for the two (a)

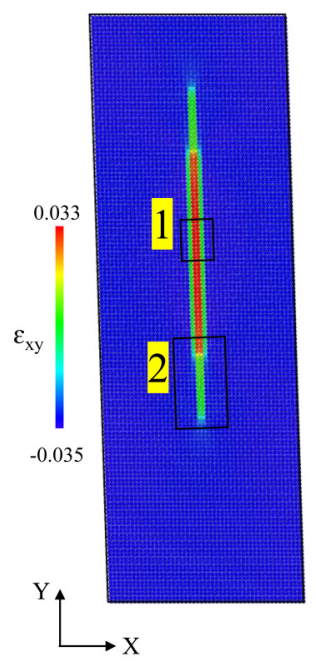

(b)

(c)

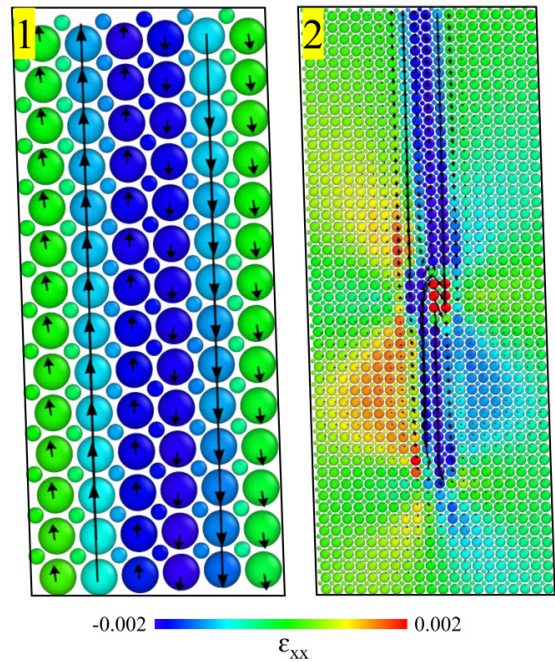

FIG. 7. (a) Double needle with two tips in a system under periodic boundary conditions. (b), (c) Atomic images with dipole vectors for site 1 and 2, respectively. The colors are coded by the atomic-level shear strain $\varepsilon_{x y}$.

outer walls. In either case we find an energy minimum for the third wall exactly in the middle. The energy difference between the middle position and the slightly shifted position (position 2 and 3 ) is very small. We find energy shifts in the order of magnitude of $0.1 \mathrm{meV} /$ atom for $d=8$ and $0.001 \mathrm{meV} /$ atom for $d=30$. This small energy explains why sideways movements are hard to observe experimentally when the walls are widely spaced: The energy gain is very small compared with common pinning energies of domain walls.

\section{Direct coupling between electric fields and needle domains}

We now explore whether a direct coupling between the electric field and a needle domain can advance or retract a needle. A needle domain with two tips at the opposite ends of the needle (a "double needle") was constructed under periodic boundary conditions [Fig. 7(a)]. The needle domains grow along the [01] direction ( $y$ axis) with a thickness of around three lattice units. The aspect ratio varies from 17.6 to 19 during the motion. Without external mechanical or electric forces this configuration is unstable because the double needle shrinks and disappears to minimize the energy. To stabilize the double needle initially, we fixed the atomic positions of the upper part of the needle and released the lower needle. This situation is similar to the pinning of a double needle at one end. The relaxed needle is shown in Fig. 7(a). The needle shaft and tip areas are colored by normal strain in the $x$ direction $\left(\varepsilon_{x x}\right)$ [Figs. 7(b) and 7(c)]. The lattice planes near the needle shaft in Fig. 7(b) contract in the $x$ direction compared with the bulk structure [the green areas in Figs. 7(b) and 7(c)] as shown for single wall. The strain field near the needle tip shows typical butterfly patterns due to the existence of kinks near the tip. Such Eshelby patterns were seen in similar simulations of kink structures in Ref. [57].

We then apply electric fields along the [10], [01], and [11] directions. To observe the needle moving due to the coupling 

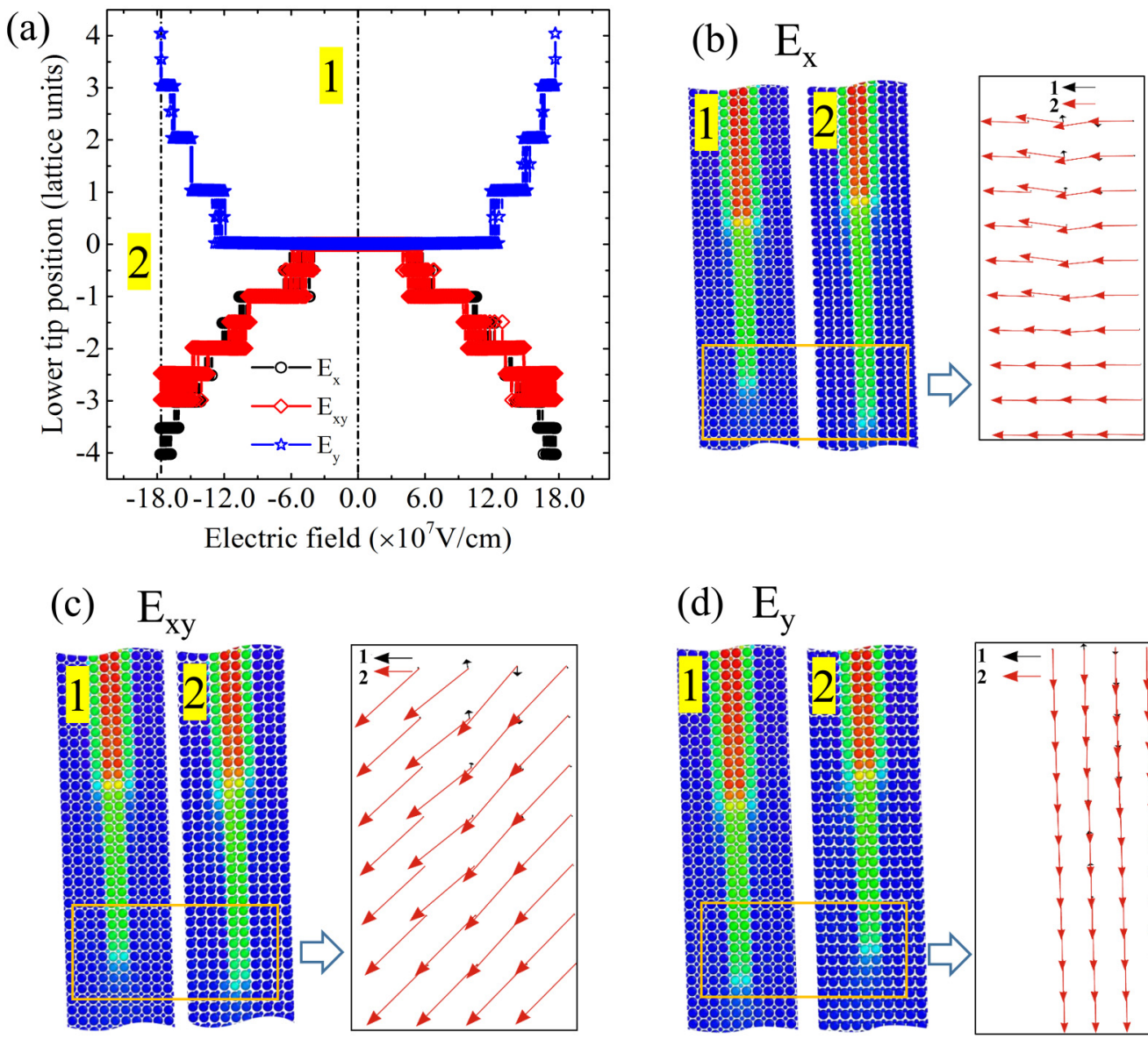

(d) $\mathrm{E}_{\mathrm{y}}$

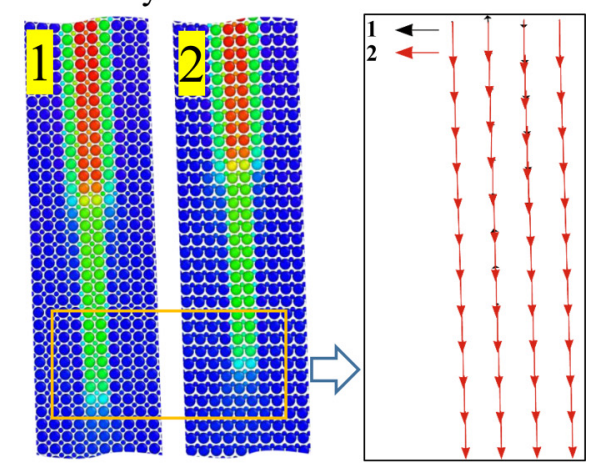

$-0.035$ 0.033

FIG. 8. The movement of needle domain in a system with periodic boundary conditions under applied electric fields. (a) The variation of tip position as a function of electric field. (b)-(d) The typical atomic images and corresponding dipole configurations under the field along [10] $\left(E_{x}\right),[11]\left(E_{x y}\right)$, and [01] $\left(E_{y}\right)$, respectively. The colors are coded by the atomic-level shear strain $\varepsilon_{x y}$.

between charge and strain, a sinusoidal electric field with amplitude $1.77 \times 10^{8} \mathrm{~V} / \mathrm{cm}$ and time repetition $1000 \tau_{\text {phonon }}$ was implemented. For fields beyond some pinning value of $|E| \approx 5 \times 10^{7} \mathrm{~V} / \mathrm{cm}$ in the [10] and [11] directions, the field moves the needle downwards by ca. $3 \sim 4$ lattice units while the electric field in the [01] direction $\left(|E|>1.3 \times 10^{8} \mathrm{~V} / \mathrm{cm}\right)$ moves the needle upwards [Fig. 8(a)]. Under periodic boundary conditions, any surface effect is excluded from the simulation. The only driving force for the moving needle stems from the rotating dipoles [Figs. 8(b)-8(d)], which change the profiles at the needle tip (wall thickness, shear angle, etc.). The needle tip, as the most active part in the needle, can be initially activated when the field is increased beyond the depinning values. The strain patterns are different for electric fields in different directions notably for needle moving upwards for a field in the [01] direction and downwards for fields in the [10] and [11] directions. Our simulations under periodic boundary conditions identify the rotations of wall dipoles to account for moving needle twins in ferroelastic materials under external electric field.

For fields beyond some pinning potential, the needle tip moves by $3 \sim 4$ lattice units. In the previous resonant piezo- electric spectroscopy (RPS) experiments [21,58], the authors found large elastic softening caused by the moving domain structures in $\mathrm{SrTiO}_{3}$. To test whether the surface makes a difference for the moving twins, we constructed a single needle under open boundary conditions (Fig. 9). The orientation of the needle domain is $45^{\circ}$ with respect to the edge of the simulation box. The thickness of the needle domain is around 3 lattice units, and aspect ratio varies between 4.6 to 9.6 during the wall motion. The free surfaces in our simulation possess an equal number of $\mathrm{A}$ and $\mathrm{B}$ atoms. The surfaces in our simulation are therefore charge-free. This configuration was previously investigated by Barrett et al. [59]. Similar to the case with periodic boundary conditions, the needle domain can easily retract to the surface to lower the energy. To retain the needle, the sample surface was fixed, the entire structure was then relaxed, the needle in the end stops at some depth below the sample surface. The dipoles inside the shaft are basically parallel to each other except for a slight tilting near the needle tip while the dipoles near the double kinks are more strongly rotated [Fig. 9(b)]. The electric fields used in this simulation are the same as with periodic boundary configuration. Electric fields along the [10], [11], and [01] 
(a)
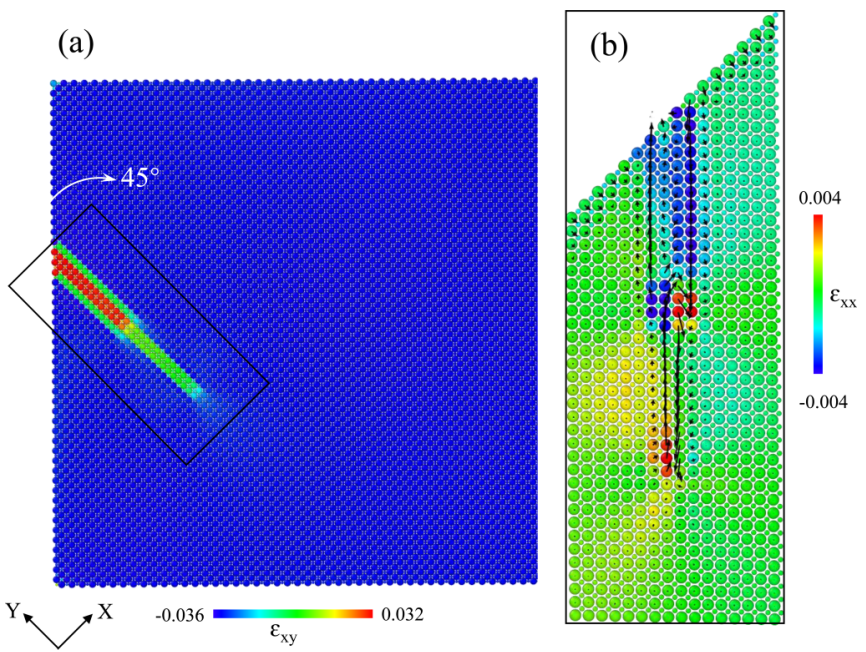

FIG. 9. (a) The configuration of a needle domain in a system with charge-free surfaces. The needle length is along the $y$-[01] direction. The colors are coded by the atomic-level shear strain $\varepsilon_{x y}$. (b) Local needle tip areas with the dipole vectors. directions were then applied. The maximum movement of the needle tip can now reach more than 15 lattice units for electric field in the [11] direction $\left(\left|E_{x}\right|=\left|E_{y}\right|=1.77 \times 10^{8} \mathrm{~V} / \mathrm{cm}\right)$.

The strain patterns near the needle tip are shown in Fig. 10. The maximum displacement is seen under maximum electric field. The tip movement extends over some 10 lattice units for field in the $x$-axis direction and in the $y$-axis direction [see Fig. 10(a)]. Thus, movement is asymmetric with positive electric field $E_{x}$ moving the needle more than the negative field [Fig. 10(a)]. The inverse holds for $E_{y}$. The maximum movement is achieved for the field in the [11] direction, when positive and negative electric fields move the needle tip. During their movement under periodic external field cycles the surface needles show periodic propagation and retraction, together with internal friction. The frictional energy loss is seen by loops near fields of $\left|E_{x}\right|=\left|E_{y}\right|=10^{7} \mathrm{~V} / \mathrm{cm}$ in Fig. 10(a). The movement under $E_{x y}$ is nearly friction free [red curves in Fig. 10(a)].

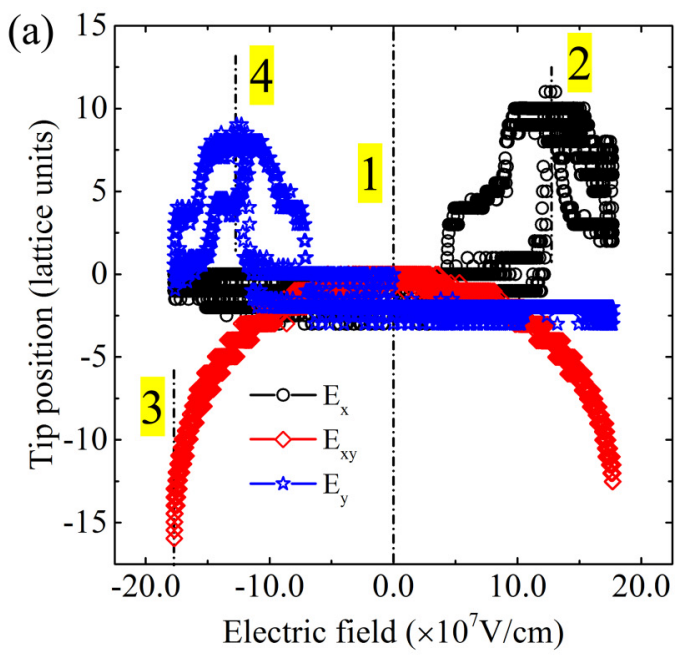

(b)

$\mathrm{E}_{\mathrm{x}}$

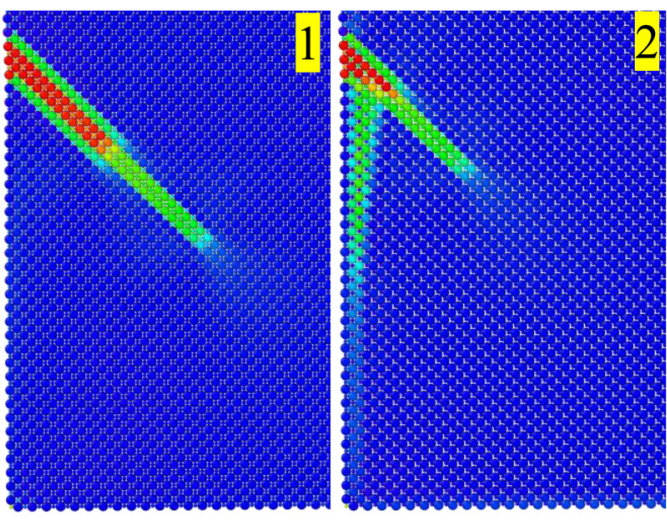

(c)

$\mathrm{E}_{\mathrm{xy}}$
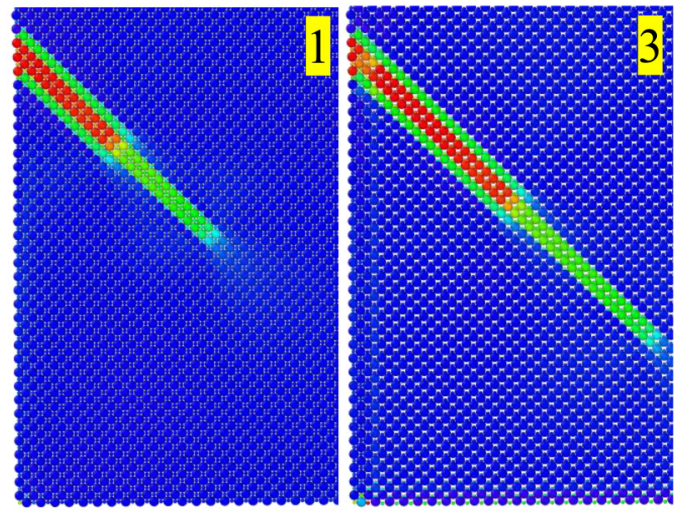

(d)

$\mathrm{E}_{\mathrm{y}}$
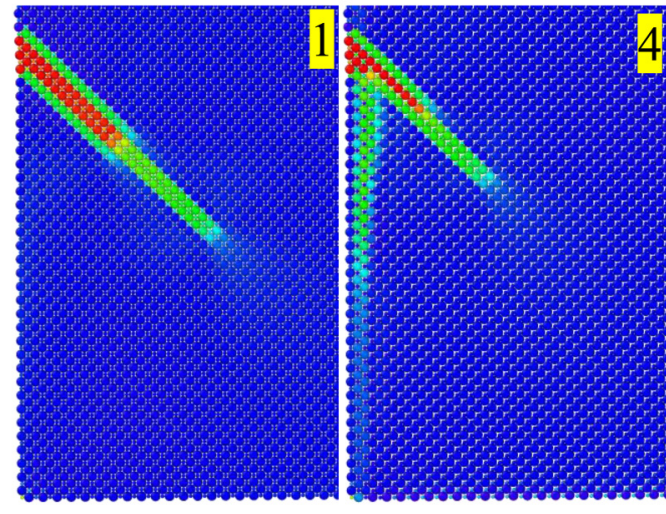

$-0.036$

$\varepsilon_{\mathrm{xy}}$

FIG. 10. The movement of needle domain in a system with charge-free surfaces under applied electric fields. (a) The variation of tip position as a function of electric field. (b)-(d) The typical atomic images under the field along [10] $\left(E_{x}\right)$, [11] $\left(E_{x y}\right)$, and [01] ( $\left.E_{y}\right)$, respectively. The colors are coded by the atomic-level shear strain $\varepsilon_{x y}$. 
(a)

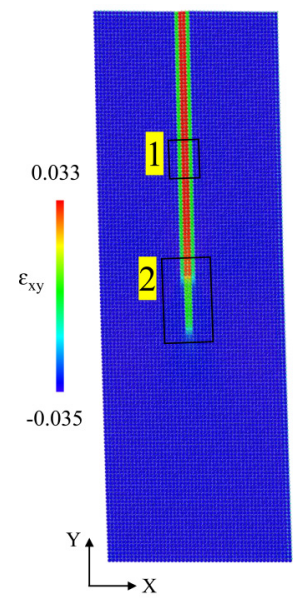

(b)

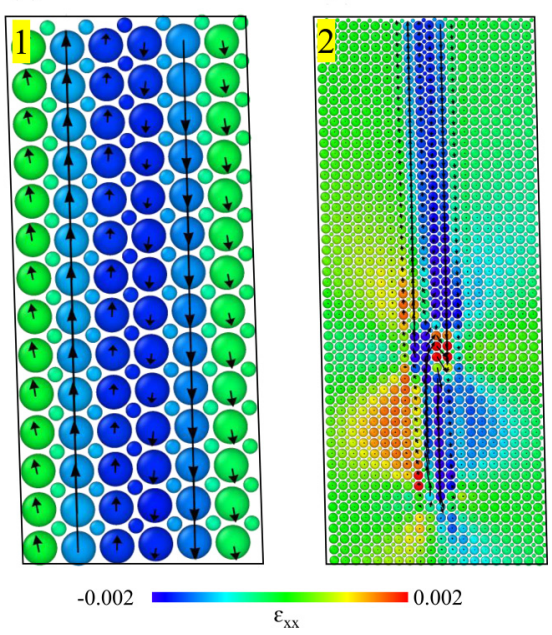

FIG. 11. (a) Needle twin structures in system with charged surface. The colors are coded by the atomic-level shear strain $\varepsilon_{x y}$. (b), (c) Atomic images with dipole vectors for site 1 and 2, respectively.

\section{E. Dancing needles in thin films with charged surfaces}

The biggest movement of the needle tip is seen when the sample surface terminations are either A or B atoms. This implies that the sample surface is charged, which may occur in thin films or in bulk material under special cleavage conditions or light illumination [50,59-61]. An applied electric field induces surface strain by attracting and repulsing the surface atoms. We make use of this configuration to enhance the field induced needle domain movement. The simulation box is shown in Fig. 11. The temperature was set to be $T=0.001 \mathrm{~K}$ and the phonon time scale was calculated to be the same as for periodic and charge-free surface systems $\left(\tau_{\text {phonon }}=3.85 \mathrm{ps}\right)$. Sinusoidal field with periodicities $\left(\tau=N \times \tau_{\text {phonon }}\right.$, where $N=0.5,1,2,5,8,10,13,20,50,100)$ were implemented with an amplitude of $1.5 \times 10^{7} \mathrm{~V} / \mathrm{cm}$. Fixing the atomic positions at the top and bottom of the sample stabilizes the initial configuration. Under these conditions the needle cannot completely retract. The thickness of the needle domain is around 3 lattice units with an aspect ratio varying from 24.6 to 36.3 during wall motion. The strain $\varepsilon_{x x}$ resonates for high frequencies with acoustic phonons. The resonance loops are shown by Fig. S2(a) [52]. As $N$ increases, the loops disappear and a phase shift between the input field and the induced strains approaches $180^{\circ}$. The length of the needle tip increases to 70 lattice units (see Fig. S2(b) in the Supplemental Material [52]) (the moving length is defined as the distance between the maximum and minimum positions of the needle tips under different $N$ ). All further simulations were then performed at a time scale $N=100$ to avoid such resonance phenomena.

The needle moves more than 35 lattice units under the sinusoidal electric field in the [11] and [01] directions and less for the field along [10] (Fig. 12). This latter direction is perpendicular to the needle direction. As the upper and lower surface layers are charged, this field could, in principle, lead to a shear movement which would advance or retract the needle tip. The two charged layers are fixed, however, to stabilize the needle so that the immobility of the needle is a consequence of this boundary condition. The atoms near the surfaces interact

(a)
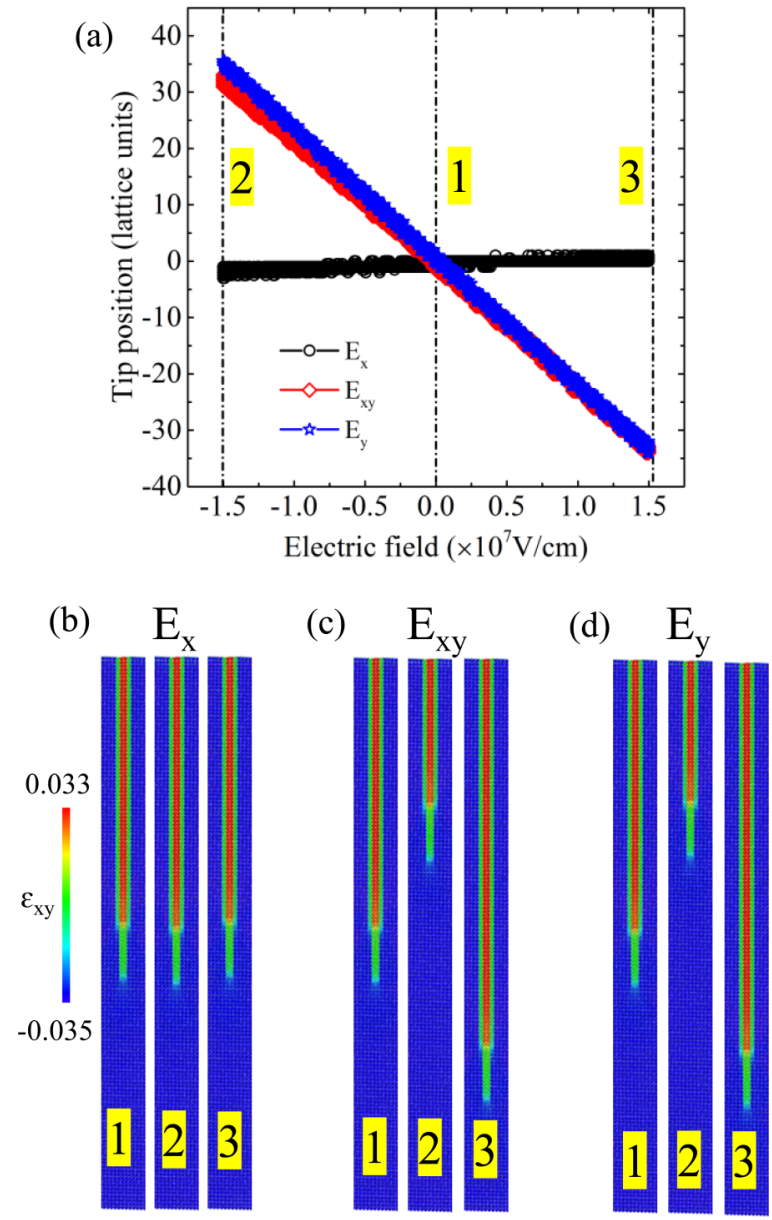

FIG. 12. (a) Moving needles in systems with charged surfaces under applied electric fields $E_{x}, E_{x y}$, and $E_{y}$. States 1,2 , and 3 refer to the system without field, with field of $-1.5 \times 10^{7} \mathrm{~V} / \mathrm{cm}$, and with field of $+1.5 \times 10^{7} \mathrm{~V} / \mathrm{cm}$, respectively. (b)-(d) The movement of needle tip moves under the field along the [10], [11], and [01] directions. For fields along the [11] and [01], the moving distance of propagation and retraction could reach around 35 lattice units.

with the external electric field, and the induced shear strain is very large compared with the previous needle strain under periodic boundary conditions or in systems without surface charges. The needle tip retracts when the applied field changes sign [see Fig. 12(a)]. The movement of the needle tip follows the field and does not show any energy loss. The needle now dances to the tune of the field. The driving forces for the moving needles are not only the direct coupling effect with the wall dipoles, but also mainly via large strain fields near the surfaces.

To clarify the different surface relaxations in these three systems, we compared the surface strains under electric field in the [11] direction $\left(E_{x}=E_{y}=1.5 \times 10^{7} \mathrm{~V} / \mathrm{cm}\right)(\mathrm{see}$ Fig. 13). The charged surface system exhibited larger surface strains [Figs. 13(a) and 13(b)] than charge-free surfaces [Figs. 13(e) and 13(f)]. The surface is absent under periodic boundary conditions [Figs. 13(c) and 13(d)], and direct coupling drives the needle movement. The field driven needle movement, hence, stems from direct coupling effect between strain and charge in needle tips (ca. $3 \sim 4$ lattice units in our 
(a) $\varepsilon_{\mathrm{xx}}$

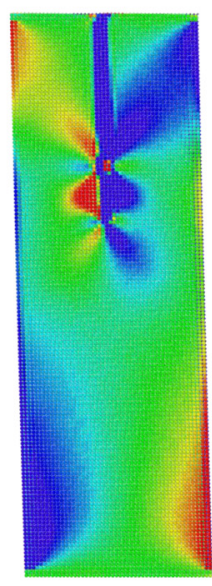

(b) $\varepsilon_{\mathrm{yy}}$

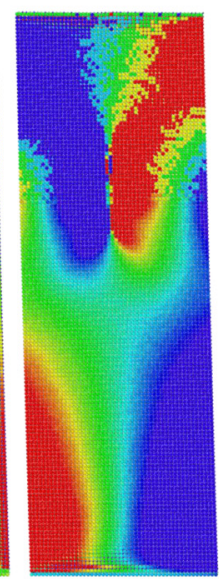

Charged surface (c) $\varepsilon_{\mathrm{xx}}$

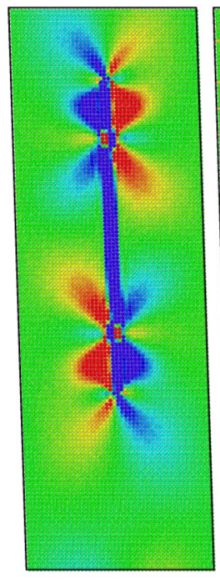

Periodic

(e) $\quad \varepsilon_{\mathrm{xx}}$

(d) $\varepsilon_{\mathrm{yy}}$
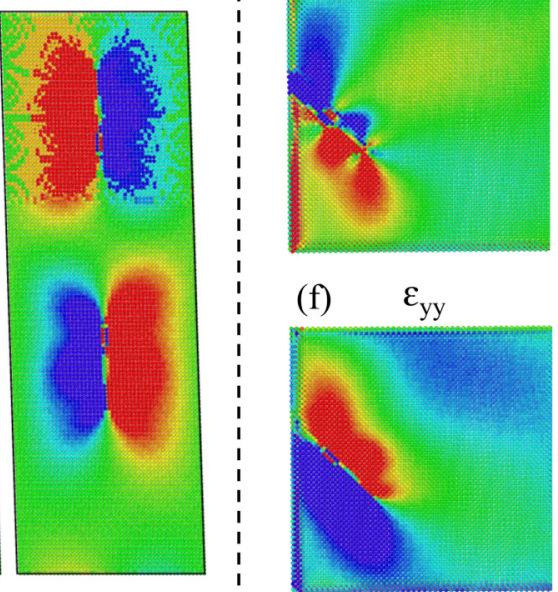

Charge-free surface

$-0.001$

0.001

FIG. 13. Comparisons of surface strains $\left(\varepsilon_{x x}\right.$ and $\left.\varepsilon_{y y}\right)$ induced by external electric field in charged surfaces (a), (b), periodic (c), (d), and charge-free surfaces (e), (f). The surface relaxations under external electric field $\left(E_{x}=E_{y}=1.5 \times 10^{7} \mathrm{~V} / \mathrm{cm}\right)$ in charged surface system is stronger than that with charge-free surfaces. No surface relaxation exists under periodic boundary conditions, where very small moving distance (3 4 lattice units) was observed.

model), the charge-free surface can provide a stronger driving forces to move the needle via additional surface relaxations. Our charge-free surface configuration under sinusoidal electric field mimics the scenario in RPS experiment, where a large elastic softening was observed due to the moving twin structures [26]. The surface relaxation in the system with charged surfaces supply the biggest driving forces compared with the charge-free surface and periodic boundary scenarios.

\section{CONCLUSION}

The retraction of needle domains over larger temperature intervals is commonly observed [16,31,62] and has been explained in terms of the elastic properties of the ferroelastic domain structures [11,12]. Moreover, the collective response of such ferroelastic domain patterns displays hallmarks for a glassy behavior as in case of low temperature relaxations during friction experiments [46] with similarities to patterns in magnetic spiral systems [63]. The main issue is whether these very sensitive reactions to external forcing or the interaction between domains in the ferroelastic phase are purely elastic in nature or whether wall polarity plays a role [23,32,64,65].

Here we considered only flexoelectric effects and find that while these effects are small, their effect on the pattern formation is appreciable. Several features, like the narrowing of domain walls under electric fields and the periodicity of several domain walls in comb configurations are a direct consequence of the local dipole moments in domain walls. A more complex behavior is found for the progression and retraction of domain walls driven by an electric field. While this effect is extremely large under strain fields, the electric field effect is small unless it generates strain effects near surfaces, grain boundaries and interfaces. The induced strain then drives the domain walls very efficiently while the direct coupling of the wall dipoles with the field is much more moderate. We have clearly identified the local wall dipoles as the key structural element, which determines the dynamics of domain pattern. This effect overrides the simple strain-strain coupling between domain walls.

\section{ACKNOWLEDGMENTS}

We are grateful to NSFC (Grants No. 51320105014 and No. 51621063) and the 111 Project (Grant No. BP 2018008) for financial support. E.K.H.S. is grateful to EPSRC (Grant No. EP/P024904/1) for support. S.L. appreciates the support from the NKRDPC (Grant No. 2019YFA0307900). G.L. is supported by a scholarship from the China Scholarship Council.
[1] E. K. H. Salje, Ann. Rev. Mater. Res. 42, 265 (2012).

[2] K. Bhattacharya, Microstructure of Martensite: Why It Forms and How It Gives Rise to the Shape-Memory Effect (Oxford University Press, Oxford, 2003), Vol. 2.
[3] R. J. Harrison, S. A. T. Redfern, and E. K. H. Salje, Phys. Rev. B 69, 144101 (2004).

[4] K. Kim, J. Korean Inst. Met. Ma. 57, 38 (2018).

[5] J. C. Agar et al., Nat. Mater. 15, 549 (2016). 
[6] E. K. H. Salje, X. Ding, Z. Zhao, T. Lookman, and A. Saxena, Phys. Rev. B 83, 104109 (2011).

[7] P. Gao et al., Nat. Commun. 5, 3801 (2014).

[8] R. J. Harrison, S. A. T. Redfern, A. Buckley, and E. K. H. Salje, J. Appl. Phys. 95, 1706 (2004).

[9] U. Bismayer, J. Hensler, E. Salje, and B. Güttler, Phase Transit. 48, 149 (1994).

[10] A. M. Bratkovsky, V. Heine, and E. K. H. Salje, Philos. T. R. Soc. A 354, 2875 (1996).

[11] E. K. H. Salje and Y. Ishibashi, J. Phys.: Condens. Matter 8, 8477 (1996)

[12] E. K. H. Salje, A. Buckley, G. van Tendeloo, Y. Ishibashi, and G. L. Nord, Am. Mineral. 83, 811 (1998).

[13] Z. Zhao, X. Ding, J. Sun, and E. K. H. Salje, J. Phys.: Condens. Matter 26, 142201 (2014).

[14] R. J. Harrison and E. K. H. Salje, Appl. Phys. Lett. 99, 151915 (2011).

[15] E. K. H. Salje, X. Wang, X. Ding, and J. Sun, Phys. Rev. B 90, 064103 (2014).

[16] R. J. Harrison and E. K. H. Salje, Appl. Phys. Lett. 97, 021907 (2010).

[17] S. Puchberger, V. Soprunyuk, W. Schranz, A. Tröster, K. Roleder, A. Majchrowski, M. A. Carpenter, and E. K. H. Salje, APL Mater. 5, 046102 (2017).

[18] X. He, S. Li, X. Ding, J. Sun, S. M. Selbach, and E. K. H. Salje, Acta Mater. 178, 26 (2019).

[19] S. Van Aert, S. Turner, R. Delville, D. Schryvers, G. Van Tendeloo, and E. K. H. Salje, Adv. Mater. 24, 523 (2012).

[20] Y. Frenkel et al., Nat. Mater. 16, 1203 (2017).

[21] L. Goncalves-Ferreira, S. A. T. Redfern, E. Atacho, and E. K. H. Salje, Appl. Phys. Lett. 94, 081903 (2009).

[22] H. Yokota, S. Matsumoto, E. K. H. Salje, and Y. Uesu, Phys. Rev. B 100, 024101 (2019).

[23] G. F. Nataf et al., Phys. Rev. Mater. 1, 074410 (2017).

[24] A. S. Yurkov and A. K. Tagantsev, Appl. Phys. Lett. 108, 022904 (2016).

[25] E. K. H. Salje, S. Li, M. Stengel, P. Gumbsch, and X. Ding, Phys. Rev. B 94, 024114 (2016).

[26] E. K. H. Salje, O. Aktas, M. A. Carpenter, V. V. Laguta, and J. F. Scott, Phys. Rev. Lett. 111, 247603 (2013).

[27] H. Yokota, H. Usami, R. Haumont, P. Hicher, J. Kaneshiro, E. K. H. Salje, and Y. Uesu, Phys. Rev. B 89, 144109 (2014).

[28] W. Gao, L. You, Y. Wang, G. Yuan, Y.-H. Chu, Z. Liu, and J.-M. Liu, Adv. Electron. Mater. 3, 1600542 (2017).

[29] J. E. Rault, T. O. Menteş, A. Locatelli, and N. Barrett, Sci. Rep. 4, 6792 (2014).

[30] E. K. H. Salje, D. Xue, X. Ding, K. A. Dahmen, and J. F. Scott, Phys. Rev. Mater. 3, 014415 (2019).

[31] B. Casals, A. Schiaffino, A. Casiraghi, S. J. Hämäläinen, D. López González, S. van Dijken, M. Stengel, and G. Herranz, Phys. Rev. Lett. 120, 217601 (2018).

[32] B. Houchmandzadeh, J. Lajzerowicz, and E. Salje, J. Phys.: Condens. Matter 3, 5163 (1991).

[33] B. Houchmanzadeh, J. Lajzerowicz, and E. Salje, Phase Transit. 38, 77 (1992).

[34] P. Marton, A. Klíč, M. Paściak, and J. Hlinka, Phys. Rev. B 96, 174110 (2017).
[35] J. Hlinka, V. Stepkova, P. Marton, and P. Ondrejkovic, in Topological Structures in Ferroic Materials: Domain Walls, Vortices and Skyrmions, edited by J. Seidel (Springer International Publishing, Cham, 2016), p. 161.

[36] J. Hlinka and P. Márton, Phys. Rev. B 74, 104104 (2006).

[37] J. M. Ball and E. C. M. Crooks, Calc. Var. Partial Differ. Equ. 40, 501 (2011)

[38] S. Conti, S. Müller, A. Poliakovsky, and E. K. H. Salje, J. Phys.: Condens. Matter 23, 142203 (2011).

[39] H. Pöttker and E. K. H. Salje, J. Phys.: Condens. Matter 26, 342201 (2014).

[40] E. K. H. Salje, Ferroelectrics 104, 111 (1990).

[41] A. M. Bratkovsky and A. P. Levanyuk, Phys. Rev. Lett. 86, 3642 (2001).

[42] A. S. Everhardt, S. Damerio, J. A. Zorn, S. Zhou, N. Domingo, G. Catalan, E. K. H. Salje, L.-Q. Chen, and B. Noheda, Phys. Rev. Lett. 123, 087603 (2019).

[43] A. E. Jacobs, Phys. Rev. B 61, 6587 (2000).

[44] E. Salje and K. Parlinski, Supercond. Sci. Technol. 4, 93 (1991).

[45] A. V. Kityk, W. Schranz, P. Sondergeld, D. Havlik, E. K. H Salje, and J. F. Scott, Phys. Rev. B 61, 946 (2000).

[46] D. Pesquera, M. A. Carpenter, and E. K. H. Salje, Phys. Rev. Lett. 121, 235701 (2018).

[47] M. Parrinello and A. Rahman, J. Appl. Phys. 52, 7182 (1981).

[48] M. Parrinello and A. Rahman, J. Chem. Phys. 76, 2662 (1982).

[49] Z. Zhao, N. Barrett, Q. Wu, D. Martinotti, L. Tortech, R. Haumont, M. Pellen, and E. K. H. Salje, Phys. Rev. Mater. 3, 043601 (2019).

[50] I. Sokolović, M. Schmid, U. Diebold, and M. Setvin, Phys. Rev. Mater. 3, 034407 (2019).

[51] L. Goncalves-Ferreira, S. A. T. Redfern, E. Artacho, and E. K. H. Salje, Phys. Rev. Lett. 101, 097602 (2008).

[52] See Supplemental Material at http://link.aps.org/supplemental/ 10.1103/PhysRevMaterials.3.114405 for additional figures.

[53] J. Novak and E. K. H. Salje, J. Phys.: Condens. Matter 10, L359 (1998).

[54] D. D. Viehland and E. K. H. Salje, Adv. Phys. 63, 267 (2014).

[55] E. K. H. Salje, S. A. Hayward, and W. T. Lee, Acta Cryst. A 61, 3 (2005).

[56] P. Barone, D. Di Sante, and S. Picozzi, Phys. Rev. B 89, 144104 (2014).

[57] E. K. H. Salje, X. Wang, X. Ding, and J. F. Scott, Adv. Funct. Mater. 27, 1700367 (2017).

[58] J. F. Scott, E. K. H. Salje, and M. A. Carpenter, Phys. Rev. Lett. 109, 187601 (2012).

[59] C. Mathieu, C. Lubin, G. Le Doueff, M. Cattelan, P. Gemeiner, B. Dkhil, E. Salje, and N. Barrett, Sci. Rep. 8, 13660 (2018).

[60] M. Saghayezhian, S. M. Rezaei Sani, J. Zhang, and E. W. Plummer, J. Phys. Chem. C 123, 8086 (2019).

[61] L. Mu, B. Zeng, X. Tao, Y. Zhao, and C. Li, J. Phys. Chem. Lett. 10, 1212 (2019).

[62] E. K. H. Salje, Z. Zhao, X. Ding, and J. Sun, Am. Mineral. 98 , 1449 (2013).

[63] S. Kustov, I. Liubimova, M. Corró, J. Torrens-Serra, X. Wang, C. R. S. Haines, and E. K. H. Salje, Sci. Rep. 9, 5076 (2019).

[64] G. Lu, S. Li, X. Ding, and E. K. H. Salje, Appl. Phys. Lett. 114, 202901 (2019).

[65] E. K. H. Salje, S. Li, Z. Zhao, P. Gumbsch, and X. Ding, Appl. Phys. Lett. 106, 212907 (2015). 